DOI: $10.26418 /$ positron.v10i2.40832

\title{
Quantum-Mechanical Brayton Engine for the Nonrelativistic Particle Trapped in a Symmetric Potential Box
}

Fikri Abdillah, Yohanes Dwi Saputra*

\author{
Physics Departement, Institut Teknologi Kalimantan, Jln. Soekarno-Hatta Km. 15, Balikpapan, Indonesia \\ *Email: yohanesngawi@lecturer.itk.ac.id \\ (Diterima 15 Mei 2020; Disetujui 14 Desember 2020; Dipublikasikan 29 Desember 2020)
}

\begin{abstract}
A theoretical quantum Brayton engine research has been carried out using a potential box system to increase its thermal efficiency. The method applied in this research is a classical thermodynamics system model in the form of a piston tube containing a monatomic ideal gas analogous to a quantum model in the form of a potential box containing one particle. The efficiency formulation of the quantum Brayton engine obtained from this study is following the classical version. However, the efficiency value obtained on a quantum Brayton engine is higher when compared to its classic. It happens because the value of the Laplace constant owned by the Brayton quantum version is 3 , while the classic version is $5 / 3$.
\end{abstract}

Keyword: compression ratio, potential box, thermal efficiency, quantum Brayton engine

\section{Introduction}

Energy is one of the basic needs that cannot be separated from human life. Geothermal, nuclear fission, and fossil fuels are a few examples of the many sources of thermal energy. In order that energy can be utilized by the public, they invented a heat engine that can convert that energy into mechanical work. However, not all heat flowed into the system is converted to mechanical work, so the rest of the heat that cannot be changed is discharged into a low-temperature bath $[1,2]$. The main problem with heat engines today is that they have low thermal efficiency. One way to increase the efficiency of a heat engine is to replace a classical system with a quantum system.

The ideal gas trapped in a piston tube is a model of the classical thermodynamics version of the system. Piston mounted on a tube can expand and compress the working substance adiabatically, isobaric, isothermal, or isochoric. When the four processes are combined, they will form cycles such as Carnot, Otto, Brayton, Diesel, etc. Then, this classical system is substituted by a quantum system. The quantum system applied can be either an infinite potential well [3-11] or a harmonic oscillator [12-14] to produce an engine efficiency that exceeds the classical version.

The adiabatic and isobaric processes are two processes that create the Brayton cycle. The adiabatic and isobaric each divided into two processes, namely the expansion and compression. In a classical system, the system absorbed thermal energy from the high-temperature bath and out of the system towards a low-temperature bath. Research on the efficiency of the quantum Brayton engine has been carried out using a 1-dimensional potential well system $[6,8,15]$. However, research on quantum heat engines using a 3-dimensional system was just being carried out by Sutantyo et al [16] and Saputra [17]. Therefore, this research offers a quantum Brayton engine with a quantum system in the form of the symmetrical 3dimensional potential well containing one particle instead of a piston tube.

\section{Theoretical Model}

The theoretical model used is to analogize a classical thermodynamics system in the form of a piston-cylinder containing monoatomic gas with a quantum system in the form of a symmetrical potential box containing a single particle. The system is represented in Figure 1.

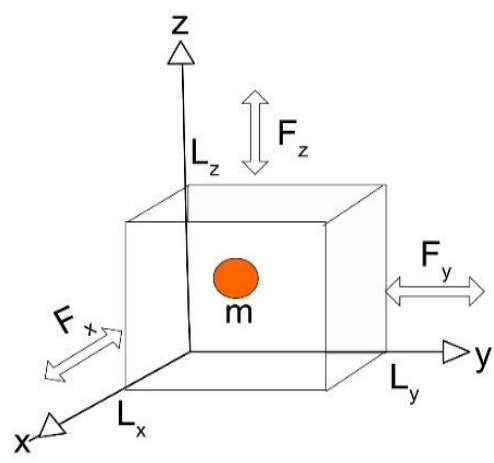

Figure 1. A particle with mass $m$ inside the potential box as a quantum system. 
That figure shows a nonrelativistic particle of mass $\mathrm{m}$ trapped in a box potential well which is symmetrical $\left(L_{x}=L_{y}=L_{z}=L\right)$. The equation that can represent the system is the independent time Schrödinger equation

$$
-\frac{\hbar^{2}}{2 m} \nabla^{2} \phi(x, y, z)=E \phi(x, y, z),
$$

with boundary condition $\phi(0, y, z)=\phi(x, 0, z)=$ $\phi(x, y, 0)=\phi(L, y, z)=\phi(x, L, z)=\phi(x, y, L)=0$, then a general solution is obtained from the equation (1) is

$$
\phi_{k, l, m}(x, y, z)=\sqrt{\frac{8}{L^{3}}} \sin \frac{k \pi x}{L} \sin \frac{l \pi y}{L} \sin \frac{m \pi z}{L},
$$

with quantum number $k, l, m=1,2,3, \ldots$ Eigenfunction in the equation (1) is associated with eigen energy

$$
E_{k l m}(L)=\left(k^{2}+l^{2}+m^{2}\right) \frac{\pi^{2} \hbar^{2}}{2 m L^{2}} .
$$

The relationship between the energy of the system in equation (3) with the mechanical force wall of the potential well is

$$
\begin{aligned}
F_{k l m}(L) & =-\frac{d E_{k l m}(L)}{d L} \\
= & \left(k^{2}+l^{2}+m^{2}\right) \frac{\pi^{2} \hbar^{2}}{m L^{3}} .
\end{aligned}
$$

Because the classical thermodynamics system is analogous to the quantum system, some thermodynamics quantities have been changed according to Bender et al [3]. Some of these quantities are shown in Table 1 . The quantum Brayton engine in this study is a quantum heat engine with a quantum system, namely a cubic potential (3D symmetric). The 3D quantum Brayton engine can be viewed as a combination of a $1 \mathrm{D}$ Brayton engine [8] whose potential walls can move freely like a piston in the $\mathrm{x}, \mathrm{y}$, and $\mathrm{z}$ (three degrees of freedom) direction. Because the system used in this study is a cubic potential $\left(L_{x}=L_{y}=L_{z}\right)$, the total work $W_{\text {total }}$ and heat input $Q_{\text {in }}$ on this 3D engine can be determined by simply calculating $W_{\text {total }}$ and $Q_{\text {in }}$ on 1D that operates on the x-axis and the result is multiplied by three. Research with a similar system for the quantum heat engine was carried out by Sutantyo et al [16] for the Carnot engine and Saputra [17] for the dual engine. By analogizing some classical thermodynamics variables to quantum version, the Brayton engine graph which is previously pressuring as a function of the volume of the cylinder $[\mathrm{P}(\mathrm{V})]$ become the mechanical force
Table 1. Quantity of the thermodynamics in the classical and quantum versions.

\begin{tabular}{|c|c|c|}
\hline No & $\begin{array}{c}\text { The Classical } \\
\text { Version }\end{array}$ & $\begin{array}{c}\text { The Quantum } \\
\text { Version }\end{array}$ \\
\hline 1 & Temperature (T) & $\begin{array}{c}\text { Expectation Value of } \\
\text { Hamiltonian or } \\
\text { Total Energy (E) }\end{array}$ \\
\hline 2 & Volume (V) & $\begin{array}{c}\text { The Width of the } \\
\text { Well (L) }\end{array}$ \\
\hline 3 & Pressure (P) & $\begin{array}{c}\text { Mechanical force on } \\
\text { the potential well } \\
\text { wall (F) }\end{array}$ \\
\hline
\end{tabular}

of the potential well wall as a function of the width of the potential well $[F(L)]$. The graph is shown in Figure 2.

However, every thermodynamic process that occurs in the reversible Brayton cycle is in a quasistatic state for a long time, so that the state of the system at each point or between two points that form a process is in a thermodynamic equilibrium condition[14]. The equation (2) and (3) are considered satisfying under this condition [17].

Point $\mathrm{A}$ is the starting point in the Brayton cycle. Because we choose that the particle is in the ground state $(k=1, l=1, m=1)$, the wave function and its total energy is $\phi_{111}$ and $E_{111}$.

We will calculate the total work $W_{\text {total }}$ and absorbed heat $Q_{\text {in }}$ for the Brayton cycle in the x-axis direction. The first stage ( $A \Leftrightarrow B$ process) is an isobaric expansion process $\left(\mathrm{L}_{A} \Leftrightarrow \mathrm{L}_{B}\right)$ in which the particle undergoes superposition in the ground state (111) to the first excitation state $(211,121$, and 112). The condition occurs because the heat flow into the system makes the particle undergo excitation. In the classical system, the heat source absorbed by the Brayton engine comes from a hightemperature bath, whereas in the quantum version it is analogous to come from a high energy bath [18]. The particle wave function $\Phi$ in this process is

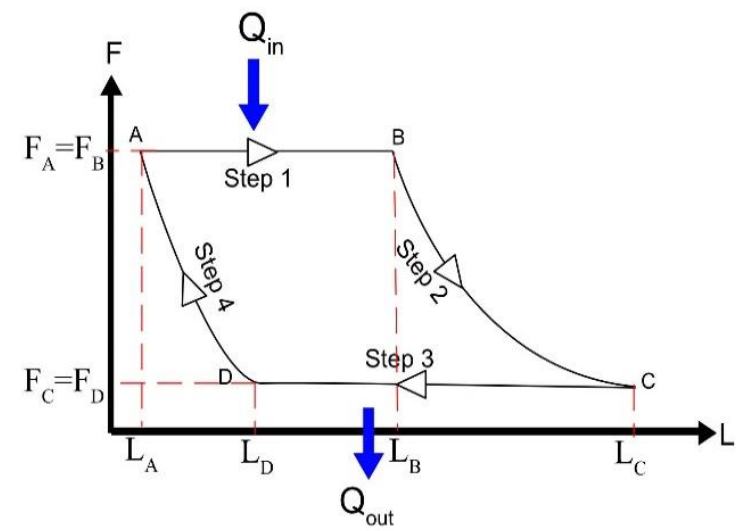

Figure 2. Quantum Brayton engine curve. 


$$
\begin{aligned}
\Phi_{A B}(x, y, z)=a_{111} \phi_{111}+a_{211} \phi_{211} \\
+a_{121} \phi_{121}+a_{112} \phi_{112} .
\end{aligned}
$$

If equation (5) is normalized, it will produce an equation

$$
\left|a_{211}\right|^{2}=\frac{1}{3}\left(1-\left|a_{111}\right|^{2}\right)
$$

The width of the wells on the $\mathrm{x}, \mathrm{y}$, and $\mathrm{z}$ axes are equal (symmetrical). So, the particle excitation state is represented by 211 levels in the $\mathrm{x}$-axis. The total energy process for a quantum system in the form of the symmetrical box is

$$
E_{A B}=\left[6-3\left|a_{111}\right|^{2}\right] \frac{\pi^{2} \hbar^{2}}{2 m L^{2}}
$$

By utilizing equation (4), the force on the first stage process is

$$
F_{A B}=\left[6-3\left|a_{111}\right|^{2}\right] \frac{\pi^{2} \hbar^{2}}{m L^{3}} .
$$

Because the first stage is the isobaric expansion, the wall of a potential box expanded in a constant force $\left(F_{A}=F_{A B}\right)$. By using equation (8) and (4) at point $\mathrm{A}$, the width of the well at point $B\left(L_{B}\right)$ is

$$
L_{B}=L_{A}(2)^{\frac{1}{3}} \text {. }
$$

By equation (9) and the mechanical force of the wall in the potential box in equation (8), then the work in the process $A \Leftrightarrow B$ that carried out by the system is

$$
W_{A B}^{(x)}=\int_{L_{A}}^{L_{B}} F_{A B} d L=\frac{3 \pi^{2} \hbar^{2}}{m L_{A}^{2}}\left[2^{\frac{1}{3}}-1\right] .
$$

By using the equation in the first law of thermodynamics, equation (10), and equation (3) applied to points $A$ and $B$, the amount of heat that enters the system is

$$
\begin{aligned}
Q_{A B}^{(x)} & =E_{B}-E_{A}+W_{A B}^{(x)} \\
& =\frac{3 \pi^{2} \hbar^{2}}{2 m}\left[\frac{3^{\frac{2}{3}} 6^{\frac{1}{3}}-3}{L_{A}^{2}}\right] .
\end{aligned}
$$

The second stage ( $B \Leftrightarrow C$ process) is an adiabatic expansion process $\left(\mathrm{L}_{B} \Leftrightarrow \mathrm{LC}\right)$. At this stage, the probability value of a particle occupying a state is fixed during the expansion process take place. Although there is no heat entering and leaving the system, the work done by the system is not zero but depends on the difference in internal energy. The particle wave function in this stage is

$$
\begin{gathered}
\Phi_{B C}(x, y, z)=a_{211} \phi_{211}+a_{121} \phi_{121} \\
+a_{112} \phi_{112} .
\end{gathered}
$$

with the system's total energy is

$$
E_{B C}(L)=\frac{6 \pi^{2} \hbar^{2}}{2 m L^{2}}
$$

By knowing the system's total energy in this process contained in the equation (13), the mechanical force of the wall in this process is

$$
F_{B C}=\frac{6 \pi^{2} \hbar^{2}}{m L^{3}}
$$

The system work in this process is

$$
W_{B C}^{(x)}=\frac{6 \pi^{2} \hbar^{2}}{2 m}\left[\frac{1}{(2)^{\frac{2}{3}} L_{A}^{2}}-\frac{1}{L_{C}^{2}}\right] .
$$

The third stage ( $C \Leftrightarrow D$ process) is an isobaric compression process $(\mathrm{LC} \Leftrightarrow \mathrm{LD})$. The particle returns to the ground state (111) because heat flows out of the system into a low energy bath during compression. The wave function $\Phi_{C D}$ and the energy $\mathrm{E}_{C D}$ in this process is the same as in equations (5) and (7). The force of the potential box wall $F_{C D}$ is the same as equation (8). The potential wall width at point $\mathrm{D}$ is

$$
L_{D}=L_{C}\left(\frac{1}{2}\right)^{\frac{1}{3}} .
$$

By using equation (8) and (16), the system work in this process is

$$
W_{C D}^{(x)}=\int_{L_{C}}^{L_{D}} F_{C D} d L=\frac{6 \pi^{2} \hbar^{2}}{2 m}\left[\frac{1}{L_{C}^{2}\left(\frac{1}{2}\right)^{\frac{2}{3}}}-\frac{1}{L_{C}^{2}}\right] .
$$

Adiabatic compression is the final process in the Brayton engine ( $D \Leftrightarrow A$ process). The state of the level occupied by the particle in this process is (111) with the wavefunction $\Phi_{D A}$ is

$$
\Phi_{D A}(x, y, z)=\phi_{111},
$$

and total energy of the system

$$
E_{D A}(L)=\frac{3 \pi^{2} \hbar^{2}}{2 m L^{2}} .
$$

with mechanical force

$$
F_{D A}(L)=\frac{3 \pi^{2} \hbar^{2}}{m L^{3}} .
$$

Mechanical work performed by the system in this process is 


$$
W_{D A}^{(x)}=\frac{3 \pi^{2} \hbar^{2}}{2 m}\left[\frac{1}{L_{A}^{2}}-\frac{1}{L_{C}^{2}\left(\frac{1}{2}\right)^{\frac{2}{3}}}\right] .
$$

By summing equation (10), (15), (17), and (21), then multiple by three, the total work for the cubic Brayton engine becomes

$$
W_{\text {total }}=\frac{9 \pi^{2} \hbar^{2}}{2 m}\left[\frac{6^{\frac{1}{3}} 3^{\frac{2}{3}}-3}{L_{A}^{2}}+\frac{6^{\frac{2}{3}} 3^{\frac{1}{3}}-6}{L_{C}^{2}}\right] .
$$

Heat absorbed by the Brayton cycle in the $\mathrm{x}, \mathrm{y}$, and $\mathrm{z}$ (3D symmetric) directions is equation (11) times three,

$$
Q_{\text {in }}=\frac{9 \pi^{2} \hbar^{2}}{2 m}\left[\frac{3^{\frac{2}{3}} 6^{\frac{1}{3}}-3}{L_{A}^{2}}\right] .
$$

The thermal efficiency of a quantum Brayton engine can be determined based on the second law of thermodynamics [1,2]. By dividing equation (22) with equation (23), then the efficiency of a quantum Brayton engine with a single particle inside the cubic potential system is

$$
\eta_{3 D}=\frac{W_{\text {total }}}{Q_{\text {in }}}=\frac{3 W_{\text {total }}^{(x)}}{3 Q_{A B}^{(x)}}=1-(2)^{\frac{2}{3}}\left(\frac{L_{A}}{L_{C}}\right)^{2} .
$$

By applying the equation (4) at point $\mathrm{A}$ and $\mathrm{C}$, the efficiency formulation (24) becomes

$$
\eta_{3 D}\left(r_{F}\right)=1-\left(\frac{1}{r_{F}}\right)^{\frac{2}{3}},
$$

with $r_{F}$ is the ratio between the force at point A with point $\mathrm{C}\left(r_{F}=\frac{F_{A}}{F_{C}}\right)$.

\section{Result and Discussion}

The equation (24) and (25) have the same form as its classical version. If the ratio of force $r_{F}$ substituted into equation (25) with the variant value and the Laplace constant value for monatomic gas $\left(\gamma_{C}=\frac{5}{3}\right)$ and single nonrelativistic particle $\left(\gamma_{Q}=3\right)$, then it will form the graph in Figure 3. In the figure, the thermal efficiency of the quantum Brayton engine has a high-efficiency value compared to the classical version even though the value of the force ratio is the same. This indicates that the efficiency of the engine can be influenced by the working substance.

The Brayton engine efficiency [see equation (24)] depends not only on the compression ratio $\left(\frac{L_{A}}{L_{C}}\right)$ but also depends on the degree of freedom of a system. The system used in this study is a 3D

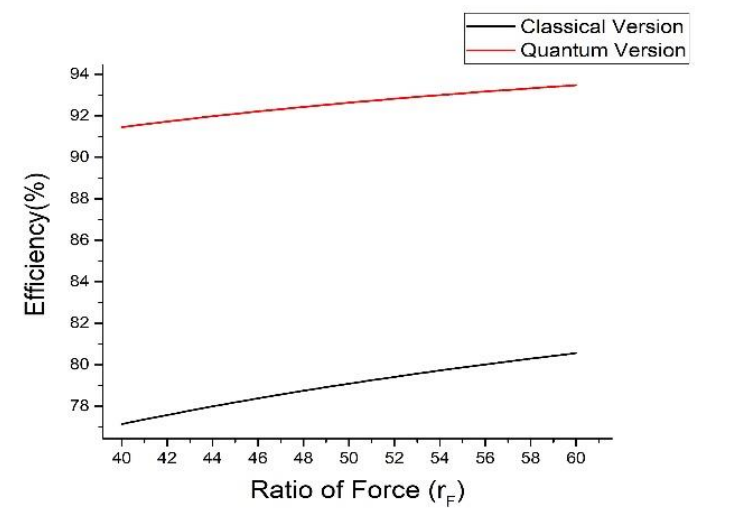

Figure 3. The efficiency curve $\eta_{3 D}\left(r_{F}\right)$ of the classical $\left(\gamma_{C}=\frac{5}{3}\right)$ and the quantum version $\left(\gamma_{Q}=\right.$ 3 ) of the Brayton engine.

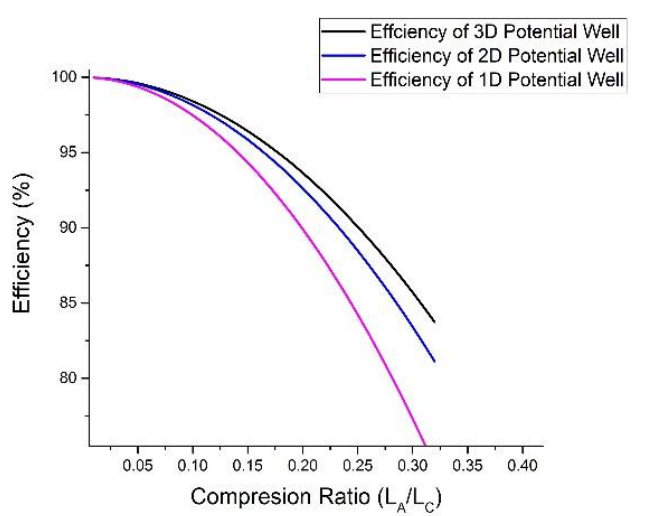

Figure 4. The efficiency curve for 1D, 2D, and 3D potential well depend on compression ratio $\left(\frac{L_{A}}{L_{C}}\right)$. potential well containing one particle. This system has three degrees of freedom represented by three levels of state, namely $k, l$, and $m$. In a previous study, the system used is a 1D with one degree of freedom [8] and 2D with two degrees of freedom [19] represented by one and two-level of state, respectively.

The efficiency equations of a quantum Brayton engine with the potential well system with different dimensions can be seen in Table 2. When the three quantum Brayton engines with the same quantum

Table 2. The equation of a quantum Brayton engine with different dimensions.

\begin{tabular}{|l|c|c|}
\hline No & $\begin{array}{c}\text { The dimension of } \\
\text { the potential well }\end{array}$ & The Efficiency \\
\hline 1 & 1D & $\eta_{1 D}=1-(4)^{\frac{2}{3}}\left(\frac{L_{A}}{L_{C}}\right)^{2}$ \\
\hline 2 & 2D & $\eta_{2 D}=1-\left(\frac{5}{2}\right)^{\frac{2}{3}}\left(\frac{L_{A}}{L_{C}}\right)^{2}$ \\
\hline 3 & 3D & $\eta_{3 D}=1-(2)^{\frac{2}{3}}\left(\frac{L_{A}}{L_{C}}\right)^{2}$ \\
\hline
\end{tabular}


system with different dimensions (1D [8], 2D [19], and 3D potential wells) are compared by substituting the same compression ratio value and the state of the particles from the ground state to the first excited state, it will produce a graph in Figure 4. If the value of the compression ratio is 0.3 , then the efficiency value of the quantum Brayton engine for 1,2 , and 3 dimensions are $77.32 \%$, $83.42 \%$, and $85.71 \%$, respectively. The efficiency of a quantum Brayton engine with a 3 dimensional potential well system shows a higher value when compared with 1D and 2D systems. This happens because the 3D system has 3 degrees of freedom.

\section{Conclusion}

Two things can be concluded in this paper. First, the thermal efficiency of the quantum Brayton engine is higher because the working substance is in the form of a single particle which has a Laplace constant $\gamma_{Q}$ value is 3 , while the classical version has a working substance in the form of monoatomic gas with a Laplace constant $\gamma_{C}$ value is $5 / 3$. The higher the Laplace constant of a working substance, the higher the efficiency of the engine [11].

Second, the efficiency with a 3D symmetrical potential well (cubic) system is higher when compared to 1D and 2D systems. The thermal efficiency of the cubic (3D) quantum system is higher than 1D and 2D due to the number of degrees of freedom it has. The 3D degree of freedom is represented by the level of state $k, l$, and $m$.

\section{Acknowledgment}

The authors thank LPPM Institut Teknologi Kalimantan for funding this research according to contract number: 2833/IT10.II/PPM.01/2020.

\section{References}

[1] Zemansky, M. W. dan Dittman, R., Heat and Thermodynamic, McGRAW-Hill Companies, Inc, 1997.

[2] Borgnakke Claus dan Sonntag, R. E., Fundamentals of Thermodynamics, John Wiley \& Sons, Inc, 2013.

[3] Bender, C. M., Brody, D. C., dan Meister, B. K., Quantum-Mechanical Carnot Engine, Journal of Physics A: Mathematical and General, 33(24), pp.4427-4436, 2000.

[4] Setyo, D. P., Latifah, E., Wisodo, H., dan Hidayat, A., Quantum Relativistic Diesel Engine with Single Massless Fermion in 1 Dimensional Box System, Jurnal Penelitian Fisika dan Aplikasinya (JPFA), 8(1), pp.25-32, 2018.
[5] Setyo, D. P. dan Latifah, E. Quantum Otto Engine based on Multiple-State Single Fermion in 1D Box System, in The 2017 International Conference on Mathematics, Science, and Education, 1093, pp. 1-7, 2018.

[6] Akbar, M. S., Latifah, E., dan Wisodo, H. Limit of Relativistic Quantum Brayton Engine of Massless Boson Trapped 1 Dimensional Potential Well, in The 2017 International Conference on Mathematics, Science, and Education, 1093, pp. 1-12, 2018.

[7] Latifah, E. dan Purwanto, A., Multiple-State Quantum Carnot Engine, Journal of Modern Physics, 2(11), pp.1366-1372, 2011.

[8] Latifah, E. dan Purwanto, A., Quantum Heat Engines; Multiple-State 1D Box System, Journal of Modern Physics, 04(08), pp.10911098, 2013.

[9] Sukamto, H., Purwanto, A., dan Subagyo, A., Mesin Panas Kuantum Partikel Relativistik pada Sumur Potensial 2 Dimensi, Jurnal Fisika dan Aplikasinya, 10(2), pp.103-107, 2014.

[10] Purwanto, A., Sukamto, H., Subagyo, B. A., dan Taufiqi, M., Two Scenarios on the Relativistic Quantum Heat Engine, Journal of Applied Mathematics and Physics, 04(07), pp.13441353, 2016.

[11] Saputra, Y. D., Quantum Lenoir Engine with a Single Particle System in a One Dimensional Infinite Potential Well, POSITRON, 9(2), pp.81-85, 2019.

[12] Yuan, Y., He, J.-Z., Yong, G., dan Wang, J.-H. Efficiency at Maximum Power Output of a Quantum-Mechanical Brayton Cycle, Communications in Theoretical Physics, 61(03), pp. 344-348, 2014.

[13] Quan, H. T., Quantum Thermodynamic Cycles and Quantum Heat Engines (II), Physical Review E, 79(4), pp. 1-10, 2009.

[14] Quan, H. T., Liu, Y., Sun, C. P., dan Nori, F., Quantum Thermodynamic Cycles and Quantum Heat Engines, Physical Review E, 76(3), pp.1-19, 2008.

[15] Singh, S., Quantum Braton Engine of NonInteracting Fermions in One-Dimensional Box, International Journal of Theoretical Physics, 59(9), pp. 2889-2900, 2020.

[16] Eka, T., Sutantyo, P., Husin, I., dan Prayitno, T., Quantum-Carnot engine for particle confined to cubic potential, in The $5^{\text {th }}$ International Conference on Mathematics and Natural Sciences., 1677, pp. 1-5., 2015.

[17] Saputra, Y. D., Quantum dual engine with working substance of a single particle inside the cubic potential, in International Conference on Science and Applied Science (ICSAS), 2296, pp. 1-8, 2020. 
[18] Bender, C. M., Brody, D. C., dan Meister, B. K., Entropy and Temperature of a Quantum Carnot Engine, in Proceeding of the Royal Society of London A: Mathematical, Physical and Engineering Sciences, 458, pp. 1519-1526, 2002.
[19] Abdillah, F., Rifani, A., dan Dwi, Y. Quantum Brayton engine based on a single particle in the 2D symmetric potential well, in Conference on Theoretical Physics and Nonlinear Phenomena (CTPNP), 2234, pp. 1-6, 2020. 